\title{
PSEUDOTUMOR INFLAMATÓRIO DO FÍGADO: DIAGNÓSTICO DIFERENCIAL DE CARCINOMA HEPATOCELULAR
}

\section{INFLAMMATORY PSEUDOTUMOR OF THE LIVER: DIFFERENTIAL DIAGNOSIS OF HEPATOCELLULAR CARCINOMA}

\author{
Antonio Nocchi Kalil - TCBC - RS ${ }^{1}$ \\ Tiago B. Franco ${ }^{2}$ \\ Roque Furian ${ }^{3}$
}

\section{INTRODUÇÃO}

O pseudotumor inflamatório do fígado (PIF) é uma lesão benigna rara, freqüentemente confundida com neoplasia maligna primária durante avaliação pré-operatória ${ }^{1,2}$. Sua patogênese é desconhecida, mas pode ser considerada uma reação tecidual benigna em resposta a um amplo espectro de agressões ${ }^{3}$.

Os achados clínicos, bem como os exames de laboratório e de imagem, não fornecem informações específicas quanto à natureza da lesão, tornando difícil seu diagnóstico diferencial pré-operatório com carcinoma hepatocelular ${ }^{1,2,4}$. Geralmente, aspiração com agulha fina demonstra uma grande quantidade de células inflamatóri$\mathrm{as}^{4}$. Portanto, a ressecção hepática é, de acordo com alguns casos relatados, o tratamento de escolha para PIF, especialmente naqueles em que o diagnóstico de certeza não é possível no pré-operatório ${ }^{3}$. Entretanto, se o diagnóstico for definido através de biopsia, e a possibilidade de neoplasia maligna esteja descartada, o tratamento conservador com corticoterapia pode ser uma alternativa adequada $^{1}$.

A possibilidade de termos tratado cirurgicamente, com sucesso, uma paciente portadora deste tipo de lesão motivou-nos a este relato.

\section{RELATO DO CASO}

Paciente branca, de 38 anos, foi admitida no Serviço de Cirurgia Geral da Santa Casa de Porto Alegre em março de 1998 com dor crônica em cólica no hipocôndrio direito, irradiando-se para o dorso e flanco homolaterais. Além disso, mencionou emagrecimento (3 quilos em 1 mês), insônia, fadiga, vômitos, diarréia e um episódio de febre $\left(39^{\circ} \mathrm{C}\right)$, o qual regredira espontaneamente. Ao exame físico, apresentava dor generalizada à palpação abdominal e o fígado era palpável abaixo do rebordo costal direito; os demais aspectos do exame físico estavam dentro da normalidade. As provas de função hepática, a dosagem da alfa-fetoproteína, a glicemia de jejum, o hemograma, a creatinina sérica, os eletrólitos séricos, a amilase e a lipase séricas eram normais. Em sua história médica pregressa, constava avaliação e realização de biopsia hepática em outro serviço, demonstrando carcinoma de células indiferenciadas, sendo, a partir de então, encaminhada ao nosso serviço.

$\mathrm{Na}$ admissão hospitalar, foi submetida à ecografia abdominal, que evidenciou um nódulo de 11,8 x 8,9 x 7,5cm no lobo hepático direito (em exame prévio, de junho de 1997, a lesão medira aproximadamente $4,5 \mathrm{~cm}$ em seu maior diâmetro), e à tomografia computadorizada abdominal que demonstrou lesão nodular no lobo hepático direito, a qual dobrara de tamanho em comparação com exame prévio realizado em agosto de 1997.

Com hipótese diagnóstica de carcinoma hepatocelular, a paciente foi submetida à hepatectomia direita (Figura 1). A análise histopatológica demonstrou pseudotumor inflamatório do fígado, com marcada proliferação de plasmócitos, tecido fibroso de granulação, neutrófilos, macrófagos e fibras colágenas (Figura 2).

A paciente teve alta hospitalar no sétimo dia pós-operatório em boas condições gerais, estando assintomática desde então.

1. Professor Adjunto da Disciplina de Cirurgia Geral da Fundação Faculdade Federal de Ciências Médicas de Porto Alegre (FFFCMPA). Chefe do Serviço de Cirurgia Oncológica do Hospital Santa Rita e do Serviço de Cirurgia Geral da Santa Casa de Porto Alegre.

2. Doutorando da FFFCMPA.

3. Professor Adjunto da Disciplina de Patologia da FFFCMPA.

Recebido em 02/04/2001.

Aceito para publicação em 22/11/2001.

Trabalho realizado no Serviço de Cirurgia Oncológica do Hospital Santa Rita e Departamento de Cirurgia da FFFCMPA/ Santa Casa de Porto Alegre. 


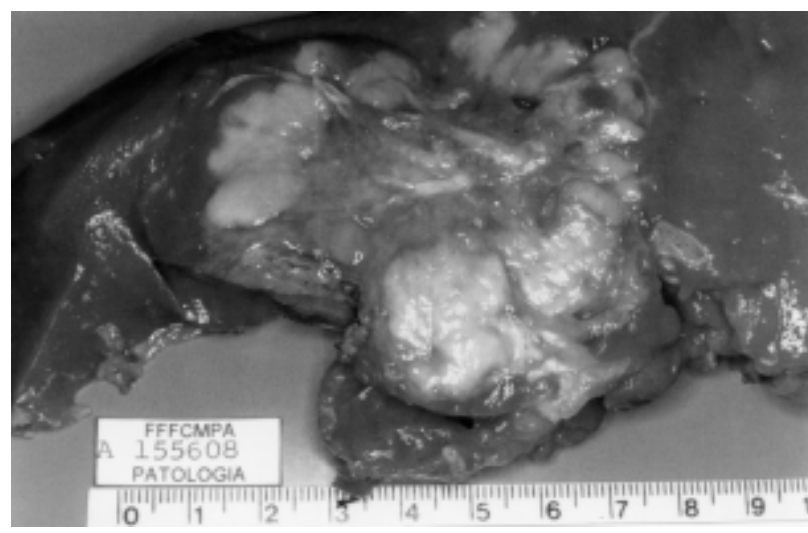

Figura 1 - Peça operatória, demonstrando lobo hepático direito com a lesão do PIF.

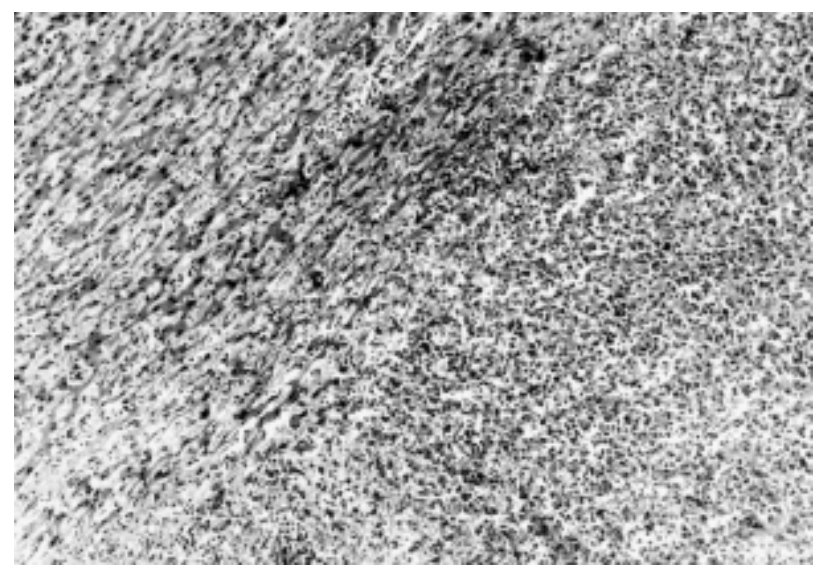

Figura 2 - Foto de exame histopatológico do PIF, demonstrando marcada proliferação de linfócitos, plasmócitos, tecido de granulação fibroso com intensa hialinização, neutrófilos, com proliferação de células fusiformes e fibras colágenas (H-E 40x).

\section{DISCUSSÃO}

O PIF pode ser definido como um grupo de doenças, cuja lesão é histologicamente semelhante à tireoidite de Riedel, fibrose retroperitoneal idiopática, fasciíte nodular e mediastinite esclerosante. Sua patogênese permanece desconhecida, mas pode ser considerada uma condição reacional inflamatória benigna. Especula-se que, entre os fa- tores causais, microrganismos ingeridos com a alimentação, infecção intra-abdominal crônica, flebite obliterante, obstrução biliar, colangite crônica, vírus Epstein-Barr, entre outros, tenham um papel em sua gênese ${ }^{5}$.

Clinicamente, a maioria dos pacientes apresenta-se com febre, dor abdominal, emagrecimento, fadiga e indisposição. Há uma tendência de leucocitose e a velocidade de sedimentação glomerular costuma estar elevada ${ }^{1,2,3}$. Baseado nos achados clínicos e laboratoriais, as hipóteses diagnósticas podem ser apontadas para uma origem infecciosa da doença ${ }^{2,3}$. O aparecimento de icterícia obstrutiva é incomum, exceto quando há envolvimento do sistema porta-hepático ou quando existe envolvimento de ambos os lobos hepáticos².

A importância clínica do PIF está no diagnóstico diferencial com carcinoma hepatocelular, 1,2,3 uma vez que os achados à ecografia, à tomografia e à ressonância nuclear magnética abdominal são semelhantes, podendo levar a equívoco diagnóstico no pré-operatório, como ocorreu no presente caso. O diagnóstico diferencial do PIF com os tumores de células fusiformes, tais como leiomiossarcoma, histiocitoma fibroso maligno e angiossarcoma, é difícil, podendo confundir o PIF com lesões malignas ${ }^{1}$. Além disso, em algumas situações, a biopsia hepática pode ser inconclusiva, demonstrando apenas tecido conjuntivo fibroso sem qualquer evidência sugestiva de malignidade, ${ }^{5}$ fato que torna duvidosa a realização da biopsia, uma vez que não mudará a conduta terapêutica. Portanto, quando o diagnóstico definitivo pré-operatório não é possível ou quando a biopsia é inespecífica, a ressecção cirúrgica deve ser realizada ${ }^{1,3}$. Por outro lado, quando o diagnóstico préoperatório é definido com certeza e naqueles casos em que a localização da lesão ou o estado clinico do paciente não permitem uma ressecção hepática segura, a corticoterapia sistêmica pode ser satisfatória 5 .

O prognóstico dos pacientes com PIF geralmente é favorável, sendo que em nenhum dos casos relatados na literatura houve óbito, obtendo-se cura após a ressecção sem complicações pós-operatórias significativas, ${ }^{3}$ conforme o relatado no presente caso, no qual a paciente apresenta-se assintomática desde a ressecção cirúrgica do PIF.

Desse modo, os autores reforçam a importância do diagnóstico diferencial entre PIF e carcinoma hepatocelular, bem como outras afecções malignas, a fim de que ao paciente seja oferecida a opção terapêutica que melhor possa contribuir para sua sobrevida e qualidade de vida.

\begin{abstract}
The authors present a rare case of inflammatory pseudotumor of the liver whose differential diagnosis with hepatocellular carcinoma - in some cases - is only possible with the histological examination. In this case, a female patient, 38 years-old was suffering from abdominal pain, enlarged liver, thinning, tiredness and fever; alpha-fetoprotein, the liver function tests, amylase and lipase were normal. Abdominal ultrasound and computed tomography showed a node in the right liver lobe. The patient was submitted to a right hepatectomy for hepatocellular carcinoma. Histological examination showed an inflammatory pseudotumor of the liver. The patient was discharged from the hospital on the seventh postoperative day; she is asymptomatic for three years.
\end{abstract}

Key words: Inflammatory pseudotumor of the liver; Hepatocellular carcinoma; Benign liver tumor. 


\section{REFERÊNCIAS}

1. Schmid A, Janig D, Bohuszlavizki A, et al. Inflammatory pseudotumor of the liver presenting as incidentaloma: report of a case and review of the literature. HepatoGastroenterol 1996; 43: 1009-14.

2. Shek TWH, Irene OL, Chan KW. Inflammatory pseudotumor of the liver. Report of four cases and review of the literature. Am J Surg Pathol 1993; 17: 231-238.

3. Uetsuji S, Nakagawa A, Kwon AH, et al. Inflammatory pseudotumor of the liver: report of a case and review of the literature. Surg Today Jpn J Surg 1996; 26: 517 21.

4. Mathiak G, Meyer PU, Mathiak M, et al. Inflammatory pseudotumor of the liver - rare differential diagnosis of undetermined hepatic space-occupying lesion. Case Report and review of the literature. Langenbecks Arch Chier 1996; 381: 309-17.

5. Anthony PP, Telesinghe PU. Inflammatory pseudotumor of the liver. J Clin Pathol 1986; 39: 761-68.

Endereço para correspondência

Dr. Antonio Nocchi Kalil, Rua Marcelo Gama, 924 / 2o, CEP 90540 - 041, Porto Alegre, RS, Brasil. Telefone (comercial): 51 346 2682. Telefone (residencial): 513428261

E-mail: ankalil.voy@terra.com.br 